\title{
Análisis espacial del índice hídrico: avances en la adopción de decisiones sostenibles en territorios agrícolas de Carabobo, Venezuela
}

\author{
Spatial analysis of the water index: advances in sustainable \\ decision-making in Carabobo agricultural territories, Venezuela
}

\author{
Barlin Orlando Olivares Campos ${ }^{1}$ \\ Universidad de Córdoba (UCO), Andalucía, España \\ Rafael Ángel Hernández $z^{2}$ \\ Richard Coelho Bonilla ${ }^{2}$ \\ Juan Carlos Molina Trigos ${ }^{2}$ \\ Yessica Pereira de López ${ }^{2}$ \\ Instit to Nacional de Meteorología e Hidrología (INAMEH), Venezuela
}

http://dx.doi.org/10.15359/rgac.60-1.10

\begin{abstract}
RESUMEN
El objetivo de este estudio fue analizar espacialmente el índice hídrico $\left(\mathrm{I}_{\mathrm{m}}\right)$, permitiendo agrupar el clima de acuerdo al grado de humedad. Se utilizó la metodología del Balance Hídrico Climático (BHC) en 25 estaciones pluviométricas en Carabobo, Venezuela, para un período de registro común (1969-1999). Los resultados muestran que se encontraron cuatro regiones, donde la humedad se comporta de modo diferente, los mayores excesos son al sur del estado y se constató que las estaciones climatológicas ubicadas al norte de Carabobo presentan un mayor déficit anual. Las zonas agrícolas más relevantes están representadas por un índice hídrico de (-2,16 a -18,96); lo que determina un clima Subhúmedo seco con precipitaciones anuales que oscilan entre los $921 \mathrm{~mm}$ y los $1063 \mathrm{~mm}$. Este estudio generó información vital en aquellas áreas agrícolas que requieren riego adicional o de controles con obras estructurales de drenaje para evitar que determinados cultivos, sensibles al exceso de agua, se perjudiquen.
\end{abstract}

1 Investigador. Master en Tecnología Ambiental. Doctorando del Programa Iberoamericano de Doctores en Agroalimentación de la Universidad de Córdoba (UCO), Andalucía, España. Correo: barlinolivares@gmail.com

2 Instituto Nacional de Meteorología e Hidrología (INAMEH), Gerencia de Meteorología Aplicada. Baruta, Venezuela.

Fecha de recepción: 05 de octubre de 2017

Fecha de aceptación: 05 de diciembre de 2017 
Barlin Orlando Olivares Campos, Andalucia, España, Rafael Ángel Hernández, Richard Coelho Bonilla, Juan Carlos Molina Trigos, Yessica Pereira de López. Spatial analysis of the water index: advances in sustainable decision-making in Carabobo agricultural territories, Venezuela

Palabras clave: agricultura, precipitación, climatología, geoestadística, déficit hídrico

\begin{abstract}
The objective of this study was to analyze spatially the water index (Im), allowing to group the climate according to the degree of humidity. The methodology of the Climatic Water Balance (BHC) for 25 rainfall stations in Carabobo, Venezuela, was used for a common registration period (19691999). The results show that four regions were found where the humidity behaves differently, the greatest excesses are to the south of the state and it was found that the climatological stations located in the north of Carabobo present a greater annual deficit. The most important agricultural areas are represented by a water index of $(-2.16$ to -18.96$)$; which determines a dry Subhumid climate with annual precipitations that oscillate between $921 \mathrm{~mm}$ and $1063 \mathrm{~mm}$. This study generated vital information in those agricultural areas requiring additional irrigation or controls with structural drainage works that prevent certain crops sensitive to excess water from being harmed.
\end{abstract}

Keywords: agriculture, precipitation, climatology, geostatistics, water deficit

\title{
Introducción
}

Hoy en día, los usuarios de los servicios de meteorología desean información sobre más aspectos del conjunto del sistema climático y terrestre. Bajo el contexto actual, los datos relacionados con el clima se usan para describir, representar y predecir tanto el comportamiento del conjunto del sistema climático (en particular, el efecto de la actividad del hombre en el clima) como la relación entre el clima y otros aspectos del entorno natural (OMM, 2011; Olivares et al., 2016c).

Las condiciones meteorológicas que influyen en la variabilidad climática natural, junto a la interacción de los elementos del clima, dan paso a la generación de los tipos climáticos que son parte fundamental, dentro de los factores formadores del suelo, del régimen hídrico de las cuencas hidrográficas, $\mathrm{y}$, que al interactuar con el resto de los factores físico naturales, condicionan la biodiversidad en general y la oferta climática, que requieren determinados cultivos para obtener superficies cosechadas rentables y sostenibles.

Particularmente, las actividades agrícolas en campo se ven condicionadas por los elementos climáticos, especialmente, por la temperatura y precipitación; es por ello, que conocer el régimen hídrico de una región tiene valiosas aplicaciones, orientadas a minimizar riesgos en los procesos de producción de bienes y servicios, además, de servir de base para la zonificación de cultivos, caracterización de sequías, determinación de épocas de siembra, programación de riego e identificación de necesidades 
Barlin Orlando Olivares Campos, Andalucía, España, Rafael Ángel Hernández, Richard Coelho Bonilla, Juan Carlos Molina Trigos, Yessica Pereira de López. Análisis espacial del índice hídrico: avances en la adopción de decisiones sostenibles en territorios agrícolas de Carabobo, Venezuela

de drenaje, entre otros (Ruiz-Álvarez et al., 2012; Olivares et al., 2012; Olivares et al., 2013a; Rodríguez et al., 2013; Olivares et al., 2017b).

En general, los territorios agrícolas tropicales se inscriben en un espacio dominado por la escasez de precipitaciones en una época del año, su elevada irregularidad y la falta de concordancia entre las necesidades requeridas por los cultivos (evapotranspiración potencial) y las lluvias (Olivares et al., 2017a). Por ello, las relaciones entre la lluvia y la agricultura se ilustran frecuentemente bajo la fórmula de balances hídricos, los cuales comparan la lluvia media mensual con los estimados requeridos de agua por los cultivos. Es necesario un criterio agronómico que permita conocer la diferenciación climática de las zonas y las necesidades hídricas de los cultivos en cada lugar y tiempo, para asegurar la producción y la renta del agricultor.

En función a lo señalado anteriormente, Thornthwaite (1948) propuso una metodología para que los climas se ordenen de acuerdo al grado de humedad, al tomar en cuenta las necesidades hídricas de los cultivos (evapotranspiración); y con los resultados del Balance Hídrico Climático (BHC): excesos y déficits, se determina el índice hídrico $\left(\mathrm{I}_{\mathrm{m}}\right)$, mismo que permite agrupar el clima en una de las clasificaciones más usadas a nivel mundial

El conocimiento de los déficits y excesos de agua, mediante la aplicación metodológica del BHC es de gran interés para determinar las posibilidades de los cultivos de secano y regadío en las zonas agrícolas de Venezuela, debido a que el aprovechamiento de las tierras de agricultura se realiza en su mayor parte sin conocer las características potenciales del clima, los suelos, y los requerimientos del cultivo, lo que no permite aprovechar al máximo la productividad, además, de la conservación y manejo sostenible de los recursos.

En el estado Carabobo, Venezuela, cada vez son más los productores agrícolas que deciden optimizar el uso del recurso hídrico con inversiones que permitan sobrellevar de manera óptima los períodos de déficit hídrico en la época seca. De allí, la importancia y la necesidad de generar información básica de las condiciones climáticas que, junto a los aspectos sociales y económicos, constituyen el impulso a la investigación y la innovación tecnológica para el desarrollo de actividades científicamente fundamentadas, económicamente rentables y ambientalmente sustentables en las áreas agrícolas degradadas, permitiendo obtener una mejor calidad de vida de sus habitantes. 
Barlin Orlando Olivares Campos, Andalucia, España, Rafael Ángel Hernández, Richard Coelho Bonilla, Juan Carlos Molina Trigos, Yessica Pereira de López. Spatial analysis of the water index: advances in sustainable decision-making in Carabobo agricultural territories, Venezuela

El objetivo de este estudio fue analizar espacialmente el índice hídrico en las zonas agrícolas del estado Carabobo en Venezuela, con el propósito de interpretar y aplicar información sobre el clima del pasado, para la adopción de decisiones, el desarrollo sostenible y la mejora de productos de información, predicciones y proyecciones relativas al clima. Adicionalmente, este estudio proporciona información simple, accesible y oportuna, entendiéndose como un producto de investigación que el usuario pueda comprender y aplicar fácilmente, además de ser de fácil acceso para un asesoramiento profesional con fines de seguimiento en territorios agrícolas.

\section{Área de estudio}

El estado Carabobo está localizado en la zona centro-norte del país, entre las coordenadas geográficas $09^{\circ} 48^{\prime}$ y $10^{\circ} 35^{\prime}$ de Latitud Norte con $67^{\circ} 31^{\prime}$ y $68^{\circ} 26^{\prime}$ de Longitud Oeste. Limita al norte con el Mar Caribe, al sur con los estados Cojedes y Guárico, al este con el estado Aragua y al oeste con el estado Yaracuy (Figura 1). Posee una superficie de $4651 \mathrm{Km}^{2}$, incluyendo $281 \mathrm{Km}^{2}$ del Lago de Valencia (Hernández et al., 2017); lo que representa el $0,5 \%$ del territorio nacional. De la superficie total, solo el $42,53 \%\left(1973 \mathrm{Km}^{2}\right)$ es adecuado para la actividad agrícola.

\section{Marco Metodológico}

\section{Información climática}

Se utilizaron los datos mensuales de precipitación de 25 estaciones Convencionales Pluviométricas (CP) ubicadas en el estado Carabobo, provenientes del Banco de Datos de la Gerencia de Procesamiento y Calidad de Datos del Instituto Nacional de Meteorología e Hidrología (INAMEH) (Tabla 1). 
Barlin Orlando Olivares Campos, Andalucia, España, Rafael Ángel Hernández, Richard Coelho Bonilla, Juan Carlos Molina Trigos, Yessica Pereira de López. Análisis espacial del índice hídrico: avances en la adopción de decisiones sostenibles en territorios agrícolas de Carabobo, Venezuela

Figura 1. Distribución espacial de las estaciones pluviométricas en el estado Carabobo, Venezuela.

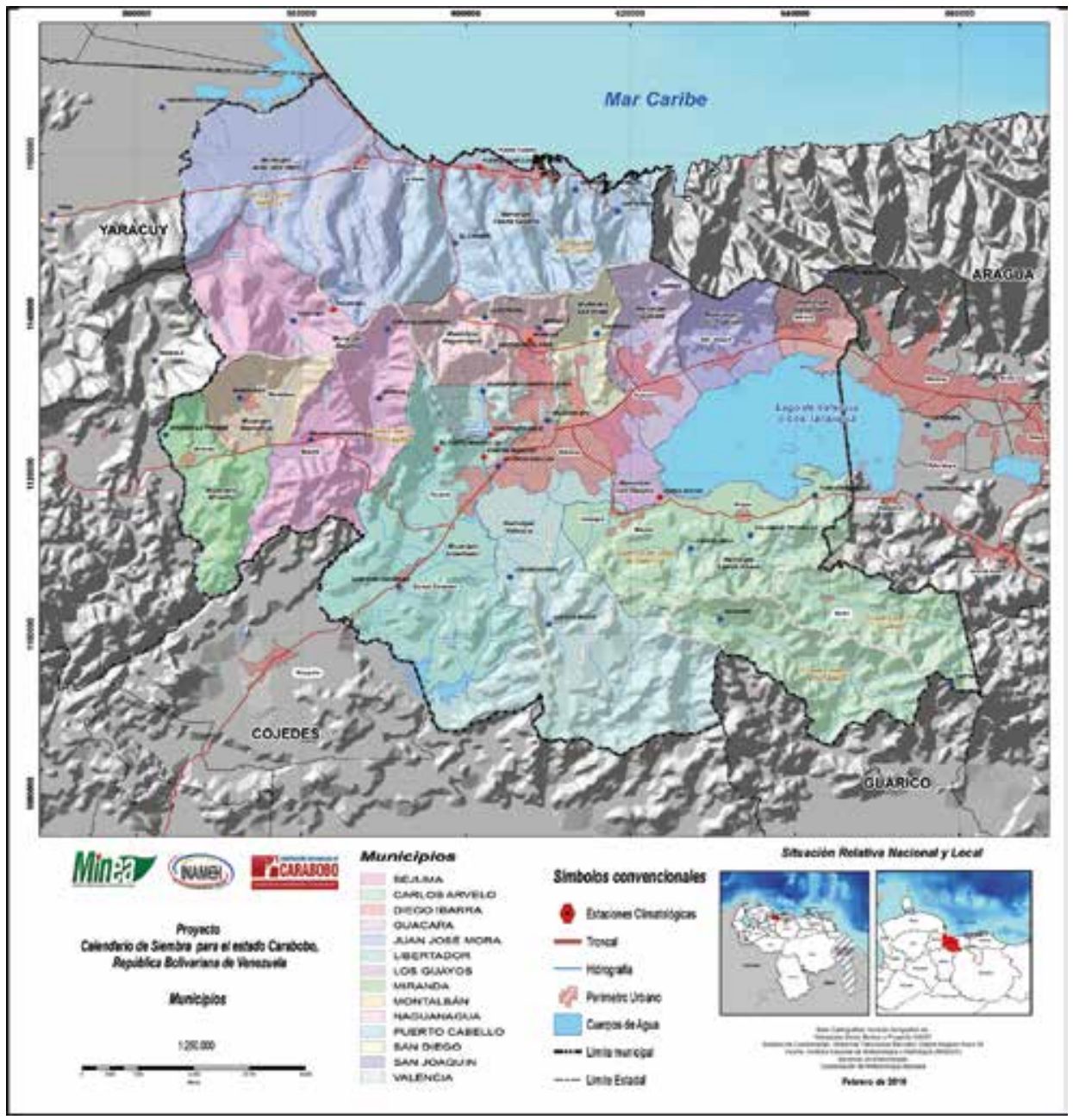

Estas estaciones se seleccionaron por poseer un período común de 30 años de registro histórico que va desde 1969 hasta 1999; este lapso de tiempo incluye el mayor número de estaciones con registros completos del estado Carabobo, de acuerdo con lo sugerido por la Organización Meteorológica Mundial (OMM, 2011). Los datos englobados se calcularon con las fórmulas de desenglobe mensual y los faltantes se estimaron a través del Método Estadístico de Regresión (Guevara, 2013). 
Barlin Orlando Olivares Campos, Andalucía, España, Rafael Ángel Hernández, Richard Coelho Bonilla, Juan Carlos Molina Trigos, Yessica Pereira de López. Spatial analysis of the water index: advances in sustainable decision-making in Carabobo agricultural territories, Venezuela

Tabla 1. Ubicación geográfica de las estaciones pluviométricas del estado Carabobo, Venezuela

\begin{tabular}{|c|c|c|c|c|c|}
\hline $\begin{array}{c}\text { Cuenca } \\
\text { hidrográfica }\end{array}$ & Estación & Municipio & Norte & Este & $\begin{array}{l}\text { Altitud } \\
\text { (msnm) }\end{array}$ \\
\hline \multirow[t]{4}{*}{ Mar Caribe } & El Cambur & Puerto Cabello & 1148809 & 598736 & 80 \\
\hline & La Entrada & Naguanagua & 1139635 & 602382 & 510 \\
\hline & Hacienda El Manglar & Puerto Cabello & 1155581 & 613403 & 20 \\
\hline & Santa Rita & Puerto Cabello & 1152833 & 618430 & 38 \\
\hline \multirow{9}{*}{$\begin{array}{l}\text { Lago de } \\
\text { Valencia }\end{array}$} & Vigirima & Guacara & 1142558 & 622845 & 557 \\
\hline & San Diego & San Diego & 1137558 & 615956 & 460 \\
\hline & Valencia-GFV & Valencia & 1126726 & 609967 & 460 \\
\hline & Yuma-Caserío & Carlos Arvelo & 1117317 & 642415 & 460 \\
\hline & Colonia El Trompillo & Carlos Arvelo & 1112434 & 634579 & 450 \\
\hline & Agua Blanca & Carlos Arvelo & 1110749 & 627339 & 515 \\
\hline & Las Dos Bocas & Valencia & 1101292 & 610166 & 550 \\
\hline & Bárbula & Naguanagua & 1136672 & 607806 & 493 \\
\hline & Valencia-San Luis & Valencia & 1120933 & 603958 & 470 \\
\hline \multirow{8}{*}{$\begin{array}{l}\text { Río } \\
\text { Portuguesa }\end{array}$} & Guataparo El Café & Naguanagua & 1135215 & 603369 & 1200 \\
\hline & Chirgua-Cabeceras & Bejuma & 1138036 & 590553 & 770 \\
\hline & Guataparo C. Inglesa & Valencia & 1130327 & 602075 & 530 \\
\hline & Montalbán & Montalbán & 1129486 & 572502 & 700 \\
\hline & Chirgua & Bejuma & 1129525 & 589480 & 740 \\
\hline & Bejuma-Panamericana & Bejuma & 1124314 & 581185 & 650 \\
\hline & Los Aguacates & Valencia & 1107115 & 605429 & 467 \\
\hline & Campo De Carabobo & Libertador & 1105972 & 591853 & 500 \\
\hline \multirow[t]{2}{*}{ Río Yaracuy } & Canoabo & Bejuma & 1139114 & 579052 & 300 \\
\hline & Miranda-La Trinidad & Miranda & 1124954 & 563534 & 628 \\
\hline Río Tiznados & Manuare & Carlos Arvelo & 1101914 & 630873 & 640 \\
\hline Río Guarico & Cartanal & Carlos Arvelo & 1093824 & 658988 & 620 \\
\hline
\end{tabular}

Fuente: Elaboración propia

Se realizó un control de calidad a los datos de precipitación mensual para definir la proporción de datos faltantes, identificar valores fuera de lo normal y observar el entendimiento básico de la distribución de las series (Parra y Cortez, 2005; Ablan et al., 2008 y Olivares et al., 2013b); utilizando los programas JMP v.6 (Jones y Sall, 2011) e InfoStat v.11 (Di Rienzo et al., 2011). 
Barlin Orlando Olivares Campos, Andalucía, España, Rafael Ángel Hernández, Richard Coelho Bonilla, Juan Carlos Molina Trigos, Yessica Pereira de López. Análisis espacial del índice hídrico: avances en la adopción de decisiones sostenibles en territorios agrícolas de Carabobo, Venezuela

Se estimó la evapotranspiración potencial (ETP) por el método FAO de Penman-Monteith, a partir de diferentes variables climáticas (Allen et al., 2006; Guevara, 2013). Este método ha sido seleccionado debido a que aproxima de una manera cercana la ETP de cualquier localidad evaluada, tiene bases físicas sólidas e incorpora explícitamente parámetros fisiológicos y aerodinámicos, y es descrito ampliamente en el estudio desarrollado por Hernández et al. (2017).

Dado que las estaciones que se disponen en el estado y sus adyacencias son en su mayoría de tipo Convencional Pluviométrica, se procedió a considerar los datos ya estimados de ETP por el Método de Penman Monteith que en su totalidad son 25 puntos a nivel mensual y anual. Dichos valores de ETP fueron correlacionados, analizados, de acuerdo al comportamiento mensual de la evaporación, y relacionados con las áreas naturales homogéneas para posteriormente ser distribuidas mediante ambiente SIG Arcview v.3.2. (ESRI, 1996) en todas las estaciones pluviométricas del estado. Todo ello con el objeto de disponer de datos meteorológicos confiables que permitan disminuir el riesgo de una sobreestimación o subestimación de la información en el cálculo de los balances hídricos.

\section{Balances Hídricos Climáticos (BHC)}

Los BHC fueron calculados para cada estación climatológica bajo un lenguaje de programación llamado Clipper (Hernández et al., 2017). El programa compila los datos de precipitación y ETP a nivel mensual; y con relación a los algoritmos de las fórmulas, ecuaciones matemáticas y estadísticas implícitas en el campo de la Agrometeorología, este realiza un procesamiento de los datos, elaborando los correspondientes balances hídricos que desglosan los valores de almacenamiento, excesos y déficit de agua en suelo, dependiendo de su profundidad y textura predominante, además de la pendiente del terreno.

\section{Índice Hídrico}

Los tipos climáticos hídricos son la expresión integral del comportamiento de un conjunto de variables a nivel mensual, tales como la precipitación, la temperatura del aire, y la evapotranspiración real y potencial, que sintetizadas en un Índice Hídrico $\left(\mathrm{I}_{\mathrm{m}}\right)$, determinan la existencia de un determinado tipo de clima y condiciones de vida existentes, potencialmente 
Barlin Orlando Olivares Campos, Andalucia, España, Rafael Ángel Hernández, Richard Coelho Bonilla, Juan Carlos Molina Trigos, Yessica Pereira de López. Spatial analysis of the water index: advances in sustainable decision-making in Carabobo agricultural territories, Venezuela

aprovechables en el campo de la siembra mediante la interrelación con los balances hídricos.

De acuerdo a esto, para el estado Carabobo se determinaron los tipos climáticos hídricos con base en la metodología propuesta por Thornthwaite (1948), detallada en la ecuación (1) y clasificaciones en la Tabla 2.

$$
I_{\mathrm{m}}=\frac{(100 * \text { Exceso })-(60 * \text { Déficit })}{E T P}
$$

Dónde: $\mathrm{E}=$ Exceso de agua durante el año;

$\mathrm{D}=$ Deficiencia de agua en el año;

$\mathrm{ETP}=$ Evapotranspiración Potencial anual.

Tabla 2. Clasificación climática en función al Índice Hídrico

\begin{tabular}{|l|c|l|}
\hline \multicolumn{1}{|c|}{ Índice Hídrico } & Símbolo & \multicolumn{1}{c|}{ Tipo Climático } \\
\hline Mayor de +100 & $\mathrm{~A}$ & Excesivamente húmedo \\
\hline$+80,1 \mathrm{a}+100$ & $\mathrm{~B} 4$ & Muy húmedo \\
\hline$+60,1 \mathrm{a}+80$ & $\mathrm{~B} 3$ & Húmedo \\
\hline$+40,1 \mathrm{a}+60$ & $\mathrm{~B} 2$ & Moderadamente húmedo \\
\hline$+20,1 \mathrm{a}+40$ & $\mathrm{~B} 1$ & Ligeramente húmedo \\
\hline$+0,1 \mathrm{a}+20$ & $\mathrm{C} 2$ & Subhúmedo húmedo \\
\hline$-19,9 \mathrm{a} 0$ & $\mathrm{C} 1$ & Subhúmedo seco \\
\hline$-39,9 \mathrm{a}-20$ & $\mathrm{D}$ & Semiárido \\
\hline Menor de -40 & $\mathrm{E}$ & Árido \\
\hline
\end{tabular}

Fuente: Thornthwaite (1948).

\section{Resultados y discusión}

Análisis de los tipos climáticos asociados al índice hídrico

Desde el punto de vista climático, el estado Carabobo se localiza entre los Climas Ecuatoriales húmedos al sur y los secos de las Altas Presiones subtropicales al norte. Los primeros deben su origen a la presencia de la Zona de Convergencia Intertropical (ZCIT), cuyo desplazamiento define el inicio de la temporada de lluvias para Venezuela; mientras que los segundos, se presentan como consecuencia de la célula anticiclónica de Azores Bermudas, que al aproximarse al ecuador terrestre en los meses de sol bajo para el hemisferio norte, contribuye a disminuir la humedad existente, generando una amplia zona de calmas climáticas, donde la precaria 
Barlin Orlando Olivares Campos, Andalucia, España, Rafael Ángel Hernández, Richard Coelho Bonilla, Juan Carlos Molina Trigos, Yessica Pereira de López. Análisis espacial del índice hídrico: avances en la adopción de decisiones sostenibles en territorios agrícolas de Carabobo, Venezuela

evolución de los sistemas nubosos, contribuye a la existencia del buen tiempo atmosférico (Hernández et al., 2017; Olivares et al., 2017b).

En el margen de estas dos estructuras, las condiciones permiten la presencia de los Climas Tropicales del estado Carabobo y el resto del país, con unos meses influenciados por la convergencia y su influencia en la génesis de precipitaciones (temporada de lluvias); y otros dominados por la ausencia de éstas (temporada seca) dentro del campo de las Altas Subtropicales del Atlántico Norte (Olivares et al., 2016a; 2016b).

De ahí, la presencia de los diversos tipos climáticos hídricos del estado Carabobo, que pueden ser clasificados en cuatro grandes grupos que de norte a sur son: Semiárido (D); Subhúmedo Seco (C1); Subhúmedo Húmedo (C2) y Ligeramente Húmedo (B1), todos con condiciones que permiten diferenciarlos tanto por su espacialidad dentro de la entidad como por la fisiografía e influencia que ésta ejerce en las condiciones climáticas existentes.

\section{Zonas del Semiárido (D)}

La superficie aproximada para este tipo climático es de $651 \mathrm{Km}^{2}$, el cual representa el 14\% del total del estado Carabobo (Figura 3); se extiende sobre los municipios Juan José Mora y Puerto Cabello en la región litoral hasta aproximadamente los $1100 \mathrm{msnm}$, y ocupa las emblemáticas regiones de Puerto Cabello, El Palito y Morón, en el área conocida como la planicie costera del centro y oeste del estado, donde paradójicamente se observa una estrecha franja litoral que colinda con el sistema montañoso de la costa y sólo cambia su configuración hacia el extremo oeste en la cuenca del Río Yaracuy, donde el paisaje pasa a ser colinar, como es el caso de Montalbán.

De acuerdo a los resultados, el $\left(\mathrm{I}_{\mathrm{m}}\right)$ para la estación Montalbán es de -21,32: lo que determina la existencia de un clima semiárido con precipitaciones anuales de $879 \mathrm{~mm}$. El balance hídrico de la estación (Figura 2a) señala un monto de precipitación anual efectiva de unos $850 \mathrm{~mm}$, con una (ETP) anual alrededor de los $1327 \mathrm{~mm}$ y supera a la precipitación durante todo el año, excepto en los meses de julio y agosto traduciéndose tal condición en la presencia de suelos secos y cursos de agua permanentes en condiciones de estiaje; en este subtipo de clima no existen excesos de agua. 
Barlin Orlando Olivares Campos, Andalucia, España, Rafael Ángel Hernández, Richard Coelho Bonilla, Juan Carlos Molina Trigos, Yessica Pereira de López. Spatial analysis of the water index: advances in sustainable decision-making in Carabobo agricultural territories, Venezuela

\section{Zonas del Subhúmedo seco (C1)}

Este tipo climático abarca una superficie de $2791,15 \mathrm{Km}^{2}$ para un $60 \%$ de la totalidad de la entidad (Figura 3); se extiende sobre los municipios Miranda, Bejuma, Naguanagua, Los Guayos, San Diego, Guácara, San Joaquín y Diego Ibarra; así como el extremo norte de los municipios Libertador, Valencia, Carlos Arvelo; y extremo sur de los municipios Juan José de Mora y Puerto Cabello. Desde el punto de vista fisiográfico, se observan amplios contrastes debido a los paisajes existentes, presentándose un relieve montañoso al norte en el eje de la Cordillera de la Costa, un paisaje colinar al suroeste en la región de Montalbán, hasta finalmente ubicarse la depresión tectónica o valle de la cuenca del Lago de Valencia en la región central.

El $\left(\mathrm{I}_{\mathrm{m}}\right)$ para esta región va desde $-2,16$ a $-18,96$; lo que determina un clima Subhúmedo seco con precipitaciones anuales que oscilan entre los $912 \mathrm{~mm}$ en la estación Cartanal (Figura 2c) y los $1033 \mathrm{~mm}$ en Chirgua Cabecera (Figura 2b). En estos sitios, la precipitación anual efectiva está alrededor de los $900 \mathrm{~mm}$, con una ETP anual de $1359 \mathrm{~mm}$ y sólo supera a la precipitación durante los meses de noviembre hasta abril, cuando los suelos se encuentran secos y los cursos de agua permanentes en condiciones de estiaje; el mes de noviembre se caracteriza por la desaparición de los excesos, pero al mismo tiempo la presencia de una importante cantidad de agua almacenada que supera los $60 \mathrm{~mm}$, reduciéndose dicho valor a medida que se acentúa la temporada seca para mostrar $0 \mathrm{~mm}$ de almacenamiento durante el mes de diciembre cuando la salida de las aguas da paso a los déficits durante los meses de enero a abril.

\section{Zonas del Subhúmedo húmedo (C2)}

Este tipo climático está emplazado sobre una superficie de 883,86 $\mathrm{Km}^{2}$ equivalentes a un $19 \%$ de la totalidad del estado; se extiende sobre el área más septentrional del municipio Valencia y meridional del municipio Libertador, así como el suroeste del municipio Carlos Arvelo, donde se encuentran las áreas agrícolas más extensas y de mayor potencial del estado Carabobo (Figura 3).

El $\left(\mathrm{I}_{\mathrm{m}}\right)$ para esta zona se ubica en el rango de 0,69 a 13,45; lo que determina la presencia de un clima Subhúmedo húmedo, donde las precipitaciones anuales oscilan en torno a los 1238,1 mm en la estación Manaure 
Barlin Orlando Olivares Campos, Andalucia, España, Rafael Ángel Hernández, Richard Coelho Bonilla, Juan Carlos Molina Trigos, Yessica Pereira de López. Análisis espacial del índice hídrico: avances en la adopción de decisiones sostenibles en territorios agrícolas de Carabobo, Venezuela

y los 1376,1 en la estación Campo Carabobo. La temporada lluviosa se extiende de mayo a octubre, la precipitación supera los índices de la ETP, generando la presencia de excesos que concentran montos superiores a los $300 \mathrm{~mm}$ anuales y que superan ampliamente la capacidad de almacenamiento de agua en los suelos (Figura 2d). El mes de abril al igual que en el tipo climático Ligeramente Húmedo, se comporta como un período transicional entre la temporada seca y la temporada lluviosa, constituyendo el escenario donde los déficits comienzan a disminuir producto del incremento de los índices de pluviosidad (Figura 2e). 
Barlin Orlando Olivares Campos, Andalucía, España, Rafael Ángel Hernández, Richard Coelho Bonilla, Juan Carlos Molina Trigos, Yessica Pereira de López. Spatial analysis of the water index: advances in sustainable decision-making in Carabobo agricultural territories, Venezuela

Figura 2. Balance hídrico para cada estación representativa (Considerando la Capacidad de Almacenamiento: $80 \mathrm{~mm}$, Textura predominante del suelo: Mediana, Profundidad: $50 \mathrm{~cm}$, Pendiente General atribuida a toda el área en estudio: $<1 \%$, Evapotranspiración Potencial por Penman-Monteith)

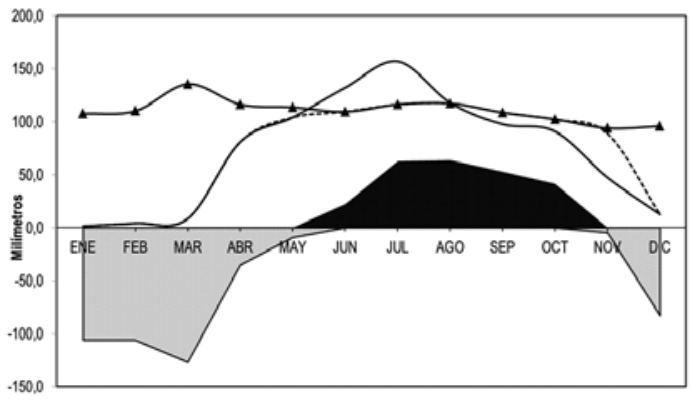

(a)

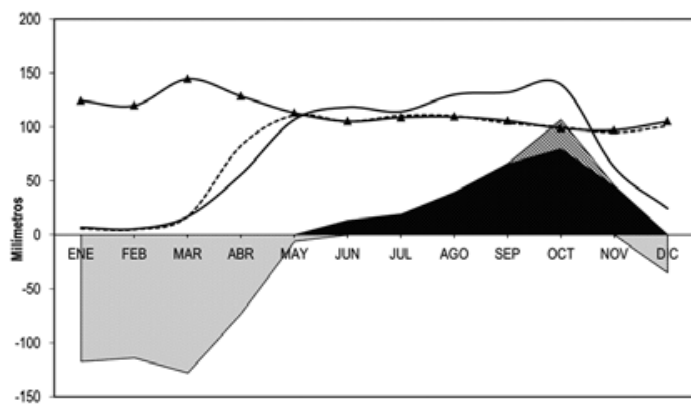

(c)

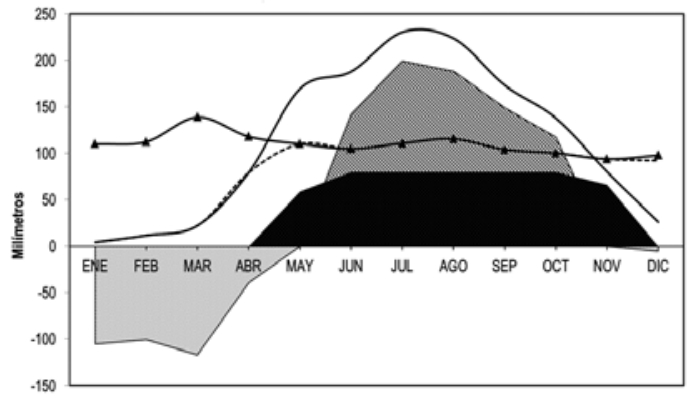

(e)

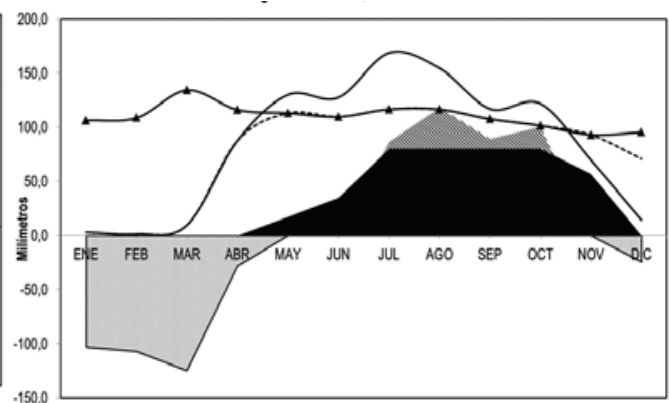

(b)

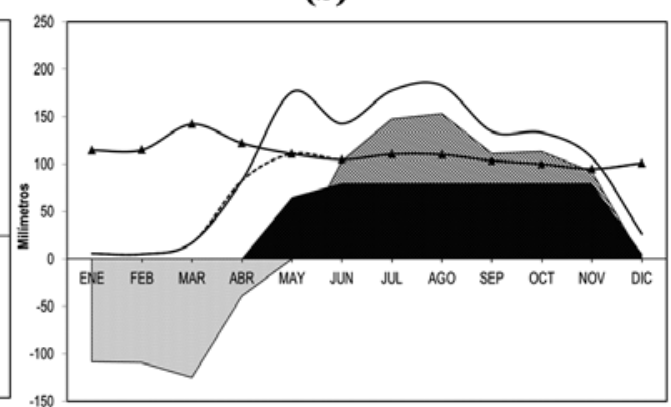

(d)

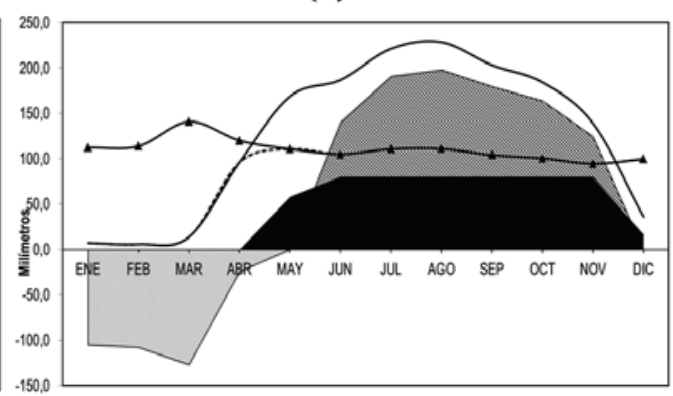

(f)
Exceso de agua -Precipitación

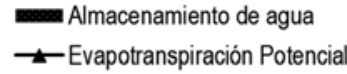

Evapotranspiración Potencia

(a) Montalbán (déficit alto); (b) Chirgua Cabecera (déficit moderado); (c) Cartanal (déficit moderado); (d) Manaure (Déficit bajo); (e) Campo Carabobo (déficit bajo); (f) Las Dos Bocas (exceso alto)

Fuente: Elaboración propia 
Barlin Orlando Olivares Campos, Andalucia, España, Rafael Ángel Hernández, Richard Coelho Bonilla, Juan Carlos Molina Trigos, Yessica Pereira de López. Análisis espacial del índice hídrico: avances en la adopción de decisiones sostenibles en territorios agrícolas de Carabobo, Venezuela

\section{Ligeramente húmedo (B1)}

Políticamente, se extiende al extremo sur del municipio Valencia hacia los límites con el estado Cojedes, ocupando una superficie de 325,63 $\mathrm{Km}^{2}$ que representa el 7\% de la totalidad del estado (Figura 3); El ( $\mathrm{I}_{\mathrm{m}}$ ) para esta región es de 22,42; lo que determina un clima ligeramente húmedo, en el que las precipitaciones anuales oscilan en torno a los $1536 \mathrm{~mm}$, de acuerdo a la información de la estación Las Dos Bocas.

El régimen de lluvias (Figura $2 \mathrm{f}$ ) muestra una distribución de tipo Unimodal con dos períodos claramente definidos; la temporada lluviosa, entre mayo a octubre, se extiende inclusive hasta noviembre, cuando se concentra el $88 \%$ de la pluviosidad, con un máximo mensual de $237 \mathrm{~mm}$ para el mes de agosto; y la temporada seca de diciembre hasta abril, cuando se concentra el $12 \%$ restante de la cantidad de agua precipitada.

Durante la temporada lluviosa, se presentan los excesos debido a que la precipitación supera los índices de la ETP, concentrándose un total de $515 \mathrm{~mm}$ acumulados desde junio a noviembre que son consecuencia de la saturación de la capacidad de almacenamiento de agua en los suelos, generando escenarios de escorrentía superficial e incremento de los niveles de agua en los ríos y quebradas.

\section{Implicaciones del déficit hídrico y excesos de agua}

Los valores de déficit derivados del cálculo de los BHC para cada una de las estaciones se presentan en la tabla 3. Los valores anuales más altos $(686 \mathrm{~mm})$ se presentan en la estación Hacienda El Manglar, distribuidos durante todo el año, seguido de Montalbán y Miranda La trinidad, con montos anuales de $470 \mathrm{~mm}$ en ambas estaciones, debido a la escaza precipitación. Es conveniente indicar que la fecha de siembra de un cultivo anual está relacionada con los rendimientos que se puedan obtener, especialmente, si depende de la precipitación como única fuente de agua. Es importante explicar el caso del maíz y algunos cereales en Carabobo, ya que los agricultores esperan las primeras lluvias para preparar el suelo y posteriormente sembrar; de modo que el cultivo aproveche el periodo de mayor precipitación para completar su periodo vegetativo y reproductivo.

En esta zona, el periodo crítico de los cultivos que normalmente se siembran, coincide con el periodo de mayor precipitación, garantizando así una buena disponibilidad de agua. En el momento de la cosecha, el 
Barlin Orlando Olivares Campos, Andalucia, España, Rafael Ángel Hernández, Richard Coelho Bonilla, Juan Carlos Molina Trigos, Yessica Pereira de López. Spatial analysis of the water index: advances in sustainable decision-making in Carabobo agricultural territories, Venezuela

periodo de lluvia está culminando, permitiendo que estos cultivos de ciclo corto completen su madurez fisiológica y que las maquinas cosechadoras y mano de obra puedan realizar la cosecha con facilidad.

En este orden de ideas, si la siembra se realiza muy temprano (abril) se corre el riesgo de que el cultivo no disponga de suficiente humedad en su periodo crítico, y, por lo tanto, sus rendimientos serán bajos, y si se siembra muy tarde entonces habrá exceso de humedad asociado con anegamiento, poca disponibilidad de oxígeno en el suelo y bajos rendimientos (Casanova, 2005; Cortez et al., 2016).

Figura 3. Distribución espacial de los tipos climáticos asociados al índice hídrico en las zonas agrícolas de Carabobo, Venezuela.

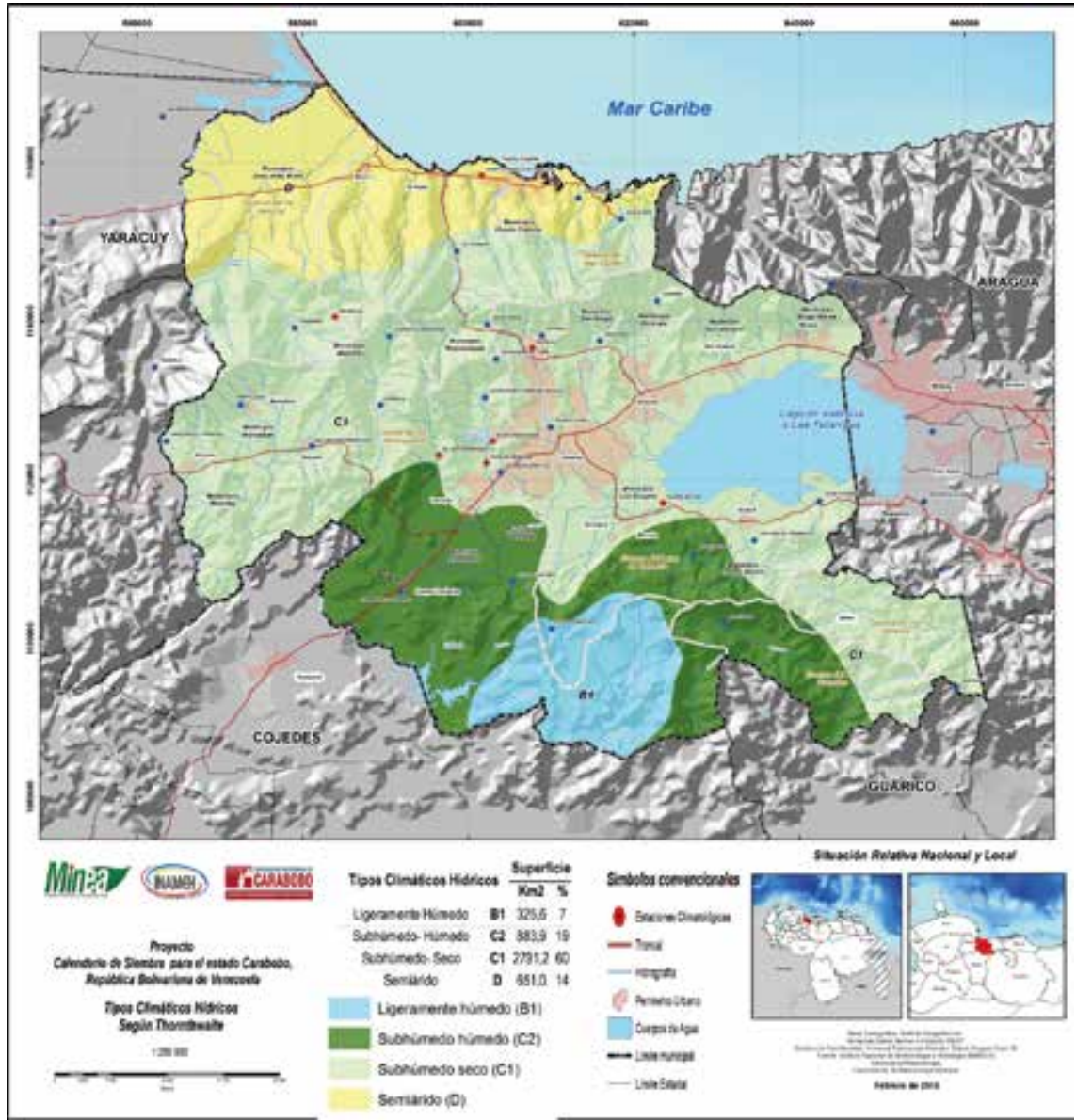


Barlin Orlando Olivares Campos, Andalucía, España, Rafael Ángel Hernández, Richard Coelho Bonilla, Juan Carlos Molina Trigos, Yessica Pereira de López. Análisis espacial del índice hídrico: avances en la adopción de decisiones sostenibles en territorios agrícolas de Carabobo, Venezuela

Es de señalar que los cultivos utilizan, para su crecimiento y funciones biológicas, apenas el $2 \%$ del agua que ingresa por sus raíces, el resto lo eliminan en forma de vapor de agua a la atmosfera, por lo que midiendo la evapotranspiración actual se tiene un valor muy aproximado de las necesidades de agua de la planta, ya que aunque utilizan un porcentaje muy bajo del agua absorbida para crecer y desarrollarse, es indispensable la circulación del resto para el reparto de los nutrientes disueltos en el agua a todo el organismo vegetal (De Ondiz, 2008).

Tabla 3. Distribución anual del déficit hídrico $(\mathrm{mm})$ para la zona de estudio.

\begin{tabular}{|c|c|c|c|c|c|c|c|c|c|c|c|c|}
\hline Estación & ene & feb & $\operatorname{mar}$ & abr & may & jun & jul & ago & sept & oct & nov & dic \\
\hline Vigirima & 103,5 & 107,6 & 129,7 & 67,6 & 0,0 & 0,0 & 0,0 & 0,0 & 0,0 & 0,0 & 0,0 & 10,4 \\
\hline San Diego & 105,3 & 108,6 & 130,1 & 64,0 & 0,0 & 0,0 & 0,0 & 0,0 & 0,0 & 0,0 & 0,0 & 34,4 \\
\hline La Entrada & 102,5 & 105,4 & 123,4 & 51,8 & 0,0 & 0,0 & 0,0 & 0,0 & 0,0 & 0,0 & 0,0 & 16,6 \\
\hline El Cambur & 85,4 & 92,1 & 120,7 & 46,6 & 14,7 & 20,3 & 0,0 & 0,0 & 0,0 & 0,0 & 0,0 & 0,0 \\
\hline Chirgua & 102,2 & 108,9 & 133,8 & 47,6 & 0,0 & 0,0 & 0,0 & 0,0 & 0,0 & 0,0 & 0,0 & 33,7 \\
\hline Montalbán & 106,0 & 106,0 & 126,8 & 35,2 & 9,4 & 0,0 & 0,0 & 0,0 & 0,0 & 0,0 & 5,0 & 83,2 \\
\hline Guataparo & 102,8 & 106,2 & 128,1 & 54,6 & 0,0 & 0,0 & 0,0 & 0,0 & 0,0 & 0,0 & 0,0 & 10,7 \\
\hline Chirgua Cabecera & 103,2 & 107,2 & 124,6 & 28,4 & 0,0 & 0,0 & 0,0 & 0,0 & 0,0 & 0,0 & 0,0 & 24,4 \\
\hline Las Dos Bocas & 105,2 & 108,0 & 127,1 & 24,0 & 0,0 & 0,0 & 0,0 & 0,0 & 0,0 & 0,0 & 0,0 & 0,0 \\
\hline Bejuma Panamericana & 102,9 & 107,9 & 125,4 & 35,0 & 0,0 & 0,0 & 0,0 & 0,0 & 0,0 & 0,0 & 0,0 & 20,9 \\
\hline Canoabo & 99,8 & 101,7 & 127,4 & 54,3 & 17,3 & 33,9 & 7,4 & 12,2 & 3,3 & 0,0 & 0,0 & 11,4 \\
\hline Miranda La Trinidad & 104,2 & 111,0 & 126,4 & 42,5 & $-2,4$ & 0,0 & 0,0 & 0,0 & 0,0 & 0,0 & 16,0 & 67,5 \\
\hline Santa Rita & 86 & 101,1 & 124,2 & 60,4 & -26 & 22,5 & 0,0 & 0,0 & 0,0 & 0,0 & 0,0 & 0,0 \\
\hline Bárbula & 104,1 & 106,8 & 130,7 & 50,7 & 0,0 & 0,0 & 0,0 & 0,0 & 0,0 & 0,0 & 0,0 & 19,8 \\
\hline Agua Blanca & 108,7 & 107,5 & 128,7 & 40,4 & 0,0 & 0,0 & 0,0 & 0,0 & 0,0 & 0,0 & 0,0 & 0,0 \\
\hline Guataparo El Café & 101,6 & 106,4 & 124,6 & 53,1 & 0,0 & 0,0 & 0,0 & 0,0 & 0,0 & 0,0 & 0,0 & 23,0 \\
\hline Hacienda EI Manglar & 89,2 & 98,9 & 117,6 & 64,9 & 57,9 & 49,8 & 20,6 & 35,8 & 45,2 & 25,8 & 33,6 & 46,9 \\
\hline Colonia EI Trompillo & 112,1 & 112,6 & 137,5 & 72,3 & 0,0 & 0,0 & 0,0 & 0,0 & 0,0 & 0,0 & 0,0 & 7,5 \\
\hline Yuma Caserio & 112,2 & 116,3 & 139,2 & 72,4 & $-2,4$ & 0,0 & 0,0 & 0,0 & 0,0 & 0,0 & 0,0 & 51,4 \\
\hline Valencia GFV & 106,3 & 109,3 & 131,3 & 46,7 & 0,0 & 0,0 & 0,0 & 0,0 & 0,0 & 0,0 & 0,0 & 36,3 \\
\hline Valencia San Luis & 107,4 & 107 & 129,1 & 38,5 & 0,0 & 0,0 & 0,0 & 0,0 & 0,0 & 0,0 & 0,0 & 30,9 \\
\hline Los Aguacates & 106,5 & 111,4 & 131 & 45,4 & 0,0 & 0,0 & 0,0 & 0,0 & 0,0 & 0,0 & 0,0 & 0 \\
\hline Campo Carabobo & 105,4 & 100,7 & 116,7 & 38,7 & 0,0 & 0,0 & 0,0 & 0,0 & 0,0 & 0,0 & 0,0 & $-5,4$ \\
\hline Manaure & 108,7 & 109,8 & 125 & 39,3 & 0,0 & 0,0 & 0,0 & 0,0 & 0,0 & 0,0 & 0,0 & 0,0 \\
\hline Cartanal & 117,4 & 114,3 & 127,9 & 72,7 & $-6,2$ & 0,0 & 0,0 & 0,0 & 0,0 & 0,0 & 0,0 & 35,3 \\
\hline
\end{tabular}

Fuente: Elaboración propia 
Barlin Orlando Olivares Campos, Andalucia, España, Rafael Ángel Hernández, Richard Coelho Bonilla, Juan Carlos Molina Trigos, Yessica Pereira de López. Spatial analysis of the water index: advances in sustainable decision-making in Carabobo agricultural territories, Venezuela

En las zonas áridas ubicadas al norte del estado Carabobo, caracterizadas por escasas precipitaciones o lluvias distribuidas en pocos meses del año, será indispensable recurrir al riego para lograr el crecimiento y fructificación de los cultivos dentro de su ciclo. El número de cosechas dependerá de la disponibilidad de agua almacenada en el embalse, por lo que la ausencia de esa obra de captación dejaría la tierra improductiva.

En aquellas zonas del estado Carabobo, especialmente, las localidades agrícolas ubicadas en el tipo climático Subhúmedo seco, que demanden riego complementario, pero tienen un periodo lluvioso definido y con extensiones que sean iguales o mayores que el ciclo de los cultivos, es posible la combinación del riego y del secano para llegar a no menos de dos cosechas por año en los cultivos de ciclo corto.

La distribución de los excesos de agua derivados de los $\mathrm{BHC}$ se muestra en la tabla 4. En las zonas al sur de Carabobo, donde llueve la mayor parte del año (estación Las Dos Bocas), se podrán tener cosechas que el ciclo de cultivo y los tiempos de preparación de tierras y de cosechas permitan, no hará falta riego complementario. Mientras más corto sea el periodo lluvioso, más agua será necesario importar, mayor será el riego complementario para poder tener una o más cosechas de un cultivo dado.

En la cuenca del lago de Valencia, gran parte de los cultivos levantados con riego no están sujetos a los vaivenes propios de la lluvia, con sequias eventuales que pueden generar pérdidas de las cosechas o con tormentas de precipitaciones intensas que dañen los cultivos por asfixia a nivel de las raíces, a menos, que se hagan cuantiosas inversiones de drenaje superficial y subsuperficial.

La regulación de las variaciones de la precipitación se logra, por lo general, con embalses que garanticen volúmenes de almacenamiento que pueden estar destinados entre otras aplicaciones al riego y al control de las inundaciones. De este modo, una sequía no será tan inclemente si se dispone de una cantidad de agua almacenada previamente para regar, o una creciente no generara tantos daños aguas abajo, si se dispone en el embalse del volumen necesario para almacenarlas (De Ondiz, 2008). 
Barlin Orlando Olivares Campos, Andalucia, España, Rafael Ángel Hernández, Richard Coelho Bonilla, Juan Carlos Molina Trigos, Yessica Pereira de López. Análisis espacial del índice hídrico: avances en la adopción de decisiones sostenibles en territorios agrícolas de Carabobo, Venezuela

Tabla 4. Distribución anual de los excesos de agua $(\mathrm{mm})$ para la zona de estudio.

\begin{tabular}{|c|c|c|c|c|c|c|c|c|c|c|c|c|}
\hline Estación & ene & feb & mar & abr & may & jun & jul & ago & sept & oct & nov & dic \\
\hline Vigirima & 0,0 & 0,0 & 0,0 & 0,0 & 0,0 & 0,0 & 0,0 & 63,6 & 30,1 & 35,5 & 0,0 & 0,0 \\
\hline San Diego & 0,0 & 0,0 & 0,0 & 0,0 & 0,0 & 0,0 & 46,2 & 39,1 & 47,9 & 18,4 & 0,0 & 0,0 \\
\hline La Entrada & 0,0 & 0,0 & 0,0 & 0,0 & 0,0 & 0,0 & 47,8 & 44,2 & 37,9 & 22,0 & 0,0 & 0,0 \\
\hline El Cambur & 0,0 & 0,0 & 0,0 & 0,0 & 0,0 & 0,0 & 0,0 & 0,0 & 0,0 & 0,0 & 0,0 & 0,0 \\
\hline Chirgua & 0,0 & 0,0 & 0,0 & 0,0 & 0,0 & 0,0 & 5,0 & 35,4 & 34,9 & 11,0 & 0,0 & 0,0 \\
\hline Montalbán & 0,0 & 0,0 & 0,0 & 0,0 & 0,0 & 0,0 & 0,0 & 0,0 & 0,0 & 0,0 & 0,0 & 0,0 \\
\hline Guataparo & 0,0 & 0,0 & 0,0 & 0,0 & 0,0 & 3,8 & 55,8 & 61,7 & 47,4 & 20,1 & 0,0 & 0,0 \\
\hline Chirgua Cabecera & 0,0 & 0,0 & 0,0 & 0,0 & 0,0 & 0,0 & 6,8 & 38,1 & 9,4 & 20,3 & 0,0 & 0,0 \\
\hline Las Dos Bocas & 0,0 & 0,0 & 0,0 & 0,0 & 0,0 & 59,9 & 110,5 & 117,5 & 99,2 & 83,7 & 44,3 & 0,0 \\
\hline Bejuma Panamericana & 0,0 & 0,0 & 0,0 & 0,0 & 0,0 & 0,0 & 38,0 & 39,0 & 6,4 & 1,3 & 0,0 & 0,0 \\
\hline Canoabo & 0,0 & 0,0 & 0,0 & 0,0 & 0,0 & 0,0 & 0,0 & 0,0 & 0,0 & 0,0 & 0,0 & 0,0 \\
\hline Miranda La Trinidad & 0,0 & 0,0 & 0,0 & 0,0 & 0,0 & 0,0 & 0,0 & 0,0 & 0,0 & 0,0 & 0,0 & 0,0 \\
\hline Santa Rita & 0,0 & 0,0 & 0,0 & 0,0 & 0,0 & 0,0 & 0,0 & 0,0 & 0,0 & 0,0 & 30,8 & 0,0 \\
\hline Bárbula & 0,0 & 0,0 & 0,0 & 0,0 & 0,0 & 0,0 & 37,7 & 60,5 & 44,3 & 24 & 0,0 & 0,0 \\
\hline Agua Blanca & 0,0 & 0,0 & 0,0 & 0,0 & 0,0 & 23,3 & 69,2 & 73,9 & 67,3 & 46,4 & 0,0 & 0,0 \\
\hline Guataparo El Café & 0,0 & 0,0 & 0,0 & 0,0 & 0,0 & 15 & 60,3 & 58,8 & 51,3 & 31,5 & 0,0 & 0,0 \\
\hline Hacienda El Manglar & 0,0 & 0,0 & 0,0 & 0,0 & 0,0 & 0,0 & 0,0 & 0,0 & 0,0 & 0,0 & 0,0 & 0,0 \\
\hline Colonia EI Trompillo & 0,0 & 0,0 & 0,0 & 0,0 & 0,0 & 0,0 & 26,2 & 49,8 & 41,3 & 15,2 & 0,0 & 0,0 \\
\hline Yuma Caserio & 0,0 & 0,0 & 0,0 & 0,0 & 0,0 & 0,0 & 0,0 & 0,0 & 47,7 & 49,5 & 0,0 & 0,0 \\
\hline Valencia GFV & 0,0 & 0,0 & 0,0 & 0,0 & 0,0 & 0,0 & 8,9 & 42,4 & 43,5 & 24,6 & 0,0 & 0,0 \\
\hline Valencia San Luis & 0,0 & 0,0 & 0,0 & 0,0 & 0,0 & 11,7 & 66,1 & 57,9 & 42,9 & 27,4 & 0,0 & 0,0 \\
\hline Los Aguacates & 0,0 & 0,0 & 0,0 & 0,0 & 0,0 & 27,3 & 102,9 & 89,7 & 83,6 & 67,9 & 6,4 & 0,0 \\
\hline Campo Carabobo & 0,0 & 0,0 & 0,0 & 0,0 & 0,0 & 62,4 & 119,6 & 108,4 & 69,3 & 37,6 & 0,0 & 0,0 \\
\hline Manaure & 0,0 & 0,0 & 0,0 & 0,0 & 0,0 & 23 & 67,2 & 72,9 & 30,8 & 33,2 & 11,8 & 0,0 \\
\hline Cartanal & 0,0 & 0,0 & 0,0 & 0,0 & 0,0 & 0,0 & 0,0 & 0,0 & 0,0 & 26,5 & 0,0 & 0,0 \\
\hline
\end{tabular}

Fuente: Elaboración propia

Para el periodo analizado, los resultados descritos evidencian que el fenómeno ENOA (El Niño-Oscilación Austral) puede explicar de forma parcial la ocurrencia de meses extremadamente secos o extremos húmedos a escala local durante las temporadas húmedas y secas en la cuenca del lago de Valencia (Paredes et al., 2014; Paredes et al., 2015; Pérez, 2012). 
Barlin Orlando Olivares Campos, Andalucia, España, Rafael Ángel Hernández, Richard Coelho Bonilla, Juan Carlos Molina Trigos, Yessica Pereira de López. Spatial analysis of the water index: advances in sustainable decision-making in Carabobo agricultural territories, Venezuela

\section{Estrategias de adaptabilidad climática}

El cambio climático observado durante las últimas décadas ha profundizado la vulnerabilidad de las poblaciones más necesitadas y los agroecosistemas de la región central de Venezuela, especialmente, en el estado Carabobo. Generalmente, parte de las zonas agrícolas padece de una crisis socioambiental derivada de la implementación de modelos extractivos y agroexportadores intensivos, así como políticas públicas con vacíos que benefician solo a una parte de la población (Ovalles et al., 2008; MARN, 2005).

La crisis hídrica en la región se ha evidenciado de manera acelerada en los últimos treinta años con la disminución de los caudales de los ríos y los nacimientos de agua, afectando sobre todo a la población rural y los pequeños agricultores, lo que ha llevado a un alto crecimiento en los precios de los bienes agrícolas, provocando, de esta manera, mayor nivel de inseguridad alimentaria.

Ante este panorama, surgen las líneas de acción y medidas de adaptación en la agricultura como actividad climáticamente sensible, enfocadas a impulsar los cambios en los sistemas de bienes alimentarios (producción y almacenaje de alimentos básicos e insumos agrícolas y su comercialización); cambios en las actividades de los sistemas de alimentos (la forma de producción, almacenaje, consumo y distribución) y por último, cambios en los componentes de seguridad alimentaria (disponibilidad, acceso, utilización y estabilidad de los alimentos).

La Tabla 5, muestra las medidas de adaptación en territorios agrícola de Carabobo, donde la capacidad de adaptación y elasticidad de la población son incrementados a través de la adopción de sistemas y prácticas productivas sustentables. 
Barlin Orlando Olivares Campos, Andalucia, España, Rafael Ángel Hernández, Richard Coelho Bonilla, Juan Carlos Molina Trigos, Yessica Pereira de López. Análisis espacial del índice hídrico: avances en la adopción de decisiones sostenibles en territorios agrícolas de Carabobo, Venezuela

Tabla 5. Medidas de adaptación climática para territorios agrícolas de secano en Carabobo, Venezuela

\begin{tabular}{|c|c|c|c|c|}
\hline $\begin{array}{c}\text { Línea de } \\
\text { acción }\end{array}$ & Ubicación & Medidas de adaptación & $\begin{array}{c}\text { Ámbito de } \\
\text { adopción }\end{array}$ & $\begin{array}{l}\text { Ámbito de } \\
\text { incidencia }\end{array}$ \\
\hline $\begin{array}{l}\text { Diversificación } \\
\text { agrícola }\end{array}$ & $\begin{array}{l}\text { Cuenca del } \\
\text { Lago de } \\
\text { Valencia y } \\
\text { Cuenca del Río } \\
\text { Portuguesa }\end{array}$ & $\begin{array}{l}\text { Rescate de especies nativas } \\
\text { Reducción de insumos agroquímicos } \\
\text { Fortalecimiento del banco de } \\
\text { germoplasma } \\
\text { Capacitación formal en prácticas } \\
\text { sustentables } \\
\text { Transferencia de tecnología } \\
\text { orientadas a las practicas sustentables } \\
\text { Promoción de colectivos dedicados } \\
\text { a la producción de semilla nativa } \\
\text { Colectivización de la tierra para } \\
\text { buenas practicas }\end{array}$ & $\begin{array}{l}\text { Familiar y local } \\
\text { por medio de } \\
\text { los colectivos } \\
\text { productores }\end{array}$ & $\begin{array}{l}\text { Municipal/ } \\
\text { sectorial }\end{array}$ \\
\hline $\begin{array}{l}\text { Gestión de las } \\
\text { cuencas más } \\
\text { vulnerables }\end{array}$ & \begin{tabular}{|l} 
Zonas altas, \\
medias y bajas \\
de las cuencas \\
hidrográficas
\end{tabular} & $\begin{array}{l}\text { Sensibilización de la población } \\
\text { en materia de conservación de los } \\
\text { recursos } \\
\text { Reforestación de la zona alta de las } \\
\text { cuencas } \\
\text { Construcción de barreras vivas }\end{array}$ & \begin{tabular}{|l} 
Organismos \\
oficiales como \\
ministerios e \\
instituciones de \\
investigación
\end{tabular} & $\begin{array}{l}\text { Estadal / } \\
\text { Regional }\end{array}$ \\
\hline $\begin{array}{l}\text { Fortalecimiento } \\
\text { de iniciativas } \\
\text { colectivas }\end{array}$ & $\begin{array}{l}\text { Cuencas: Río } \\
\text { portuguesa, Río } \\
\text { Tiznados, Río } \\
\text { Yaracuy y Lago } \\
\text { de Valencia }\end{array}$ & $\begin{array}{l}\text { Para productores dedicados a } \\
\text { la agricultura de subsistencia: } \\
\text { creación de un espacio local para la } \\
\text { comercialización de la producción } \\
\text { Para jóvenes productores: } \\
\text { promoción de la formación de } \\
\text { nuevos colectivos productores } \\
\text { Para productores de agricultura } \\
\text { convencional: fortalecimiento de } \\
\text { sus capacidades mediante procesos } \\
\text { de formación teóricos-prácticos } \\
\text { que les permitan incrementar su } \\
\text { conocimiento local, identidad } \\
\text { cultural y auto- sustentabilidad. }\end{array}$ & $\begin{array}{l}\text { Organizaciones } \\
\text { comunitarias y } \\
\text { gremiales }\end{array}$ & $\begin{array}{l}\text { Local / } \\
\text { Municipal }\end{array}$ \\
\hline
\end{tabular}

Fuente: Adaptado de Hernández et al. (2017).

\section{Conclusiones}

El estudio concentra los resultados del BHC, el cual permitió conocer la variación interanual del déficit y excesos. De esta forma, se coloca en evidencia la influencia que la variabilidad interanual del clima puede ejercer en la caracterización climática de Carabobo, y su repercusión en las áreas agrícolas. Esto permite sugerir el uso de esta metodología para 
Barlin Orlando Olivares Campos, Andalucia, España, Rafael Ángel Hernández, Richard Coelho Bonilla, Juan Carlos Molina Trigos, Yessica Pereira de López. Spatial analysis of the water index: advances in sustainable decision-making in Carabobo agricultural territories, Venezuela

obtener los valores de déficits y excesos resultantes de los BHC, con fines de planificación, zonificación y caracterización del régimen hídrico de cualquier localidad.

Considerando la magnitud del déficit hídrico en estas zona agrícolas del estado Carabobo, se debería enfrentar los episodios secos severos mediante una mayor dotación de recursos para la construcción de pozos profundos, aprovechando además causes de los ríos y quebradas cercanos; para ello, es necesario contar con un plan estratégico a nivel regional para determinar las cuencas fluviales más importantes en la zona, con el objeto de generar una reserva para potenciar el riego en el sector agropecuario.

Los resultados dejan en evidencia que la zonificación de los riesgos de déficit y excesos de agua en áreas potenciales para cultivos facilitará la creación de base de datos de apoyo al ordenamiento ecológico del estado Carabobo. Esto permitiría a largo plazo, realizar un manejo sustentable de los recursos naturales a partir de un conocimiento profundo de las potencialidades, así como su conservación con conocimiento previo.

\section{Agradecimiento}

Agradecemos el apoyo financiero de la Secretaría de Seguridad Alimentaria y Desarrollo Agrario de la Gobernación del estado Carabobo, Venezuela.

\section{Referencias}

Ablan, M., Andressen, R., Vargas, M. P. y Acevedo, M. (2008). Propuesta metodológica para el control de calidad de datos de precipitación. Agronomía Trop, 58(1), 57-60. Recuperado de http://sian.inia.gob.ve/repositorio/revistas_ci/Agronomia\%20Tropical/at5801/pdf/ablan_m.pdf

Allen, R.G., Pereira, L.S., Raes, D. y Smith, M. (2006). Evapotranspiración del cultivo. Guías para la determinación de los requerimientos de agua de los cultivos. Roma, Italia, FAO. 323 p. (Serie Estudio de Riego y Drenaje No. 56).

Casanova, E. (2005). Introducción a la ciencia del suelo. Editorial del Consejo de desarrollo científico y humanístico. UCV. Caracas, Venezuela. Cortez, A., Rodríguez, M., Rey, J.C., Ovalles, F., González, W., Parra, R., Olivares, B. y Marquina, J. (2016). Variabilidad espacio temporal de la precipitación en el estado Guárico, Venezuela. Rev. Fac. Agron (LUZ), 33 (3), 292-310. 
Barlin Orlando Olivares Campos, Andalucia, España, Rafael Ángel Hernández, Richard Coelho Bonilla, Juan Carlos Molina Trigos, Yessica Pereira de López. Análisis espacial del índice hídrico: avances en la adopción de decisiones sostenibles en territorios agrícolas de Carabobo, Venezuela

De Ondiz, J. (2008). Riego y drenaje agrícola para ingenieros. Editorial del Consejo de Desarrollo Científico y Humanístico. UCV. Caracas, Venezuela.

Di Rienzo J.A., Casanoves F., Balzarini M.G., González L., Tablada M., Robledo C.W. (2011). InfoStat versión 2011. Grupo InfoStat, FCA, Universidad Nacional de Córdoba, Argentina. Recuperado de: http:// www.infostat.com.ar

ESRI. (1996). Arc View GIS. The geographic information system for everyone. Versión 3.2. by ESRI. Product ID: 825921104087.

Guevara, J.M. (2013). Métodos de estimación y ajuste de datos climáticos. Caracas, Venezuela. Editorial del Consejo de desarrollo científico y humanístico. UCV...

Hernández, R., Pereira, Y., Molina, JC., Coelho, R., Olivares, B. y Rodríguez, K. (2017). Calendario de siembra para las zonas agrícolas del estado Carabobo en la República Bolivariana de Venezuela. Sevilla España, Editorial Universidad Internacional de Andalucía.

Jones, B. y Sall, J. (2011). JMP statistical discovery software. Wiley Interdisciplinary Reviews: Computational Statistics, 3(3), 188-194.

MARN (Ministerio del Ambiente y de los Recursos Naturales, Venezuela). (2005). Primera Comunicación Nacional en Cambio Climático de Venezuela. Programa de las Naciones Unidas para el Desarrollo (PNUD). Caracas, Venezuela.

Olivares, B., Parra, R., Cortez, A. y Rodríguez, M.F. (2012). Patrones de homogeneidad pluviométrica en estaciones climáticas del estado Anzoátegui, Venezuela. Revista Multiciencias, 12 (Extraordinario), 11-17.

Olivares B., Caraballo, L. y Torrealba, J. (2013a). Variabilidad del régimen de precipitación en el periodo 1990-2009 en la localidad de El Tigre estado Anzoátegui, Venezuela. Rev. Fac. Agron (LUZ), 30 (1), 19-32. Recuperado de http://revfacagronluz.org.ve/PDF/enero_marzo2013/v30n1a20131932.pdf

Olivares, B., Cortez, A., Parra, R., Rodríguez, M. y Guevara, E. (2013b). Aplicación de procedimientos estadísticos para el control de calidad de las series de precipitación mensual de los llanos orientales venezolanos. Rev. Fac. Agron (LUZ), 30 (3), 367-391. Recuperado de http://revfacagronluz.org.ve/PDF/julio_septiembre2013/ v30n3a2013367391.pdf 
Barlin Orlando Olivares Campos, Andalucia, España, Rafael Ángel Hernández, Richard Coelho Bonilla, Juan Carlos Molina Trigos, Yessica Pereira de López. Spatial analysis of the water index: advances in sustainable decision-making in Carabobo agricultural territories, Venezuela

Olivares, B., Cortez, A., Lobo, D., Parra, R., Rey, J.C. y Rodríguez, M. (2016a). Estudio de la Sequía Meteorológica en Localidades de los Llanos de Venezuela Mediante el Índice de Precipitación Estandarizado. Revista Acta Nova, 7 (3), 266-283.

Olivares, B., Cortez, A., Lobo, D., Parra, R., Rey, J.C. y Rodríguez, M. (2016b). Análisis temporal de la sequía meteorológica en localidades semiáridas de Venezuela. UGCiencia, 22 (1),11-24.

Olivares, B., Cortez, A., Muñetones, A. y Casana, S. (2016c). Strategic Elements of Organizational Knowledge Management for Innovation. Case: Agrometeorology Network. Revista Digital de Investigación en Docencia Universitaria, 10 (1), 68-81.

Olivares, B., Cortez, A., Lobo, D., Parra, R., Rey, J.C. y Rodríguez, M. (2017a). Evaluation of agricultural vulnerability to drought weather in different locations of Venezuela. Rev. Fac. Agron. (LUZ) 34 (1): 103-129.

Olivares, B., Zingaretti, M. L., Demey Zambrano, J.A. y Demey, J. R. (2017b). Aplicación del método STATIS-ACT al régimen de lluvias en la Región Oriental Venezolana. UNED Research Journal, 9(1), 97-106.

OMM (Organización Meteorológica Mundial, Suiza). (2011). Guía de prácticas climatológicas. OMM-N ${ }^{\circ} 100$. Ginebra. Suiza.

Ovalles, F., Cortez, A., Rodríguez, M.F., Rey, J.C. y Cabrera-Bisbal, E. (2008). Variación geográfica del impacto del cambio climático en el sector agrícola en Venezuela. Agronomía Trop, 58 (1), 37 - 40.

Paredes, F., La Cruz, F. y Guevara, E. (2014). Análisis regional de frecuencia de las sequías meteorológicas en la principal región cerealera de Venezuela. Bioagro, 26(1), 21-28.

Paredes, F., Guevara-Pérez, E., Barbosa-Alves, H. y Uzcátegui-Briceño, C. (2015). Tendencia de la precipitación estacional e influencia de El Niño-Oscilación Austral sobre la ocurrencia de extremos pluviométricos en la cuenca del lago. Tecnología y Ciencias del Agua VI (6), 33-48.

Parra, R.y Cortez, A. (2005). Control de calidad de series de precipitación de las series de precipitación del INIA Venezuela en el periodo 19702000. Rev. Arg. de Agrometeorología, (5-6), 63-73. 
Barlin Orlando Olivares Campos, Andalucia, España, Rafael Ángel Hernández, Richard Coelho Bonilla, Juan Carlos Molina Trigos, Yessica Pereira de López. Análisis espacial del índice hídrico: avances en la adopción de decisiones sostenibles en territorios agrícolas de Carabobo, Venezuela

Pérez, N. L. (2012). Influencia de episodios El Niño-Oscilación del Sur (ENOS) sobre la precipitación en el Estado Monagas, Venezuela. Revista Cientifica UDO Agricola, 12(2), 400-406.

Rodríguez, M.F., Cortez, A., Olivares, B., Rey, J.C., Parra, R. y Lobo, D. (2013). Análisis espacio temporal de la precipitación del estado Anzoátegui y sus alrededores. Agronomía Trop, 63 (1-2), 57-65.

Ruiz-Álvarez, O., Arteaga-Ramírez, R., Vázquez-Peña, M.A., Ontiveros Capurata, R.E. y López-López, R. (2012). Balance hídrico y clasificación climática del estado de Tabasco, México. Universidad y Ciencia, 28(1), 1-14.

Thornthwaite, C.W. (1948). An approach toward a rational classification of climate. American Geographical Society, 38(1), 55-94. 